\title{
Besonderheiten bei der Notfallversorgung von geriatrischen Traumapatienten
}

Stefan Barzen, Gertraude Koetter, Bernd Wohlrath, Uwe Schweigkofler, Reinhard Hoffmann
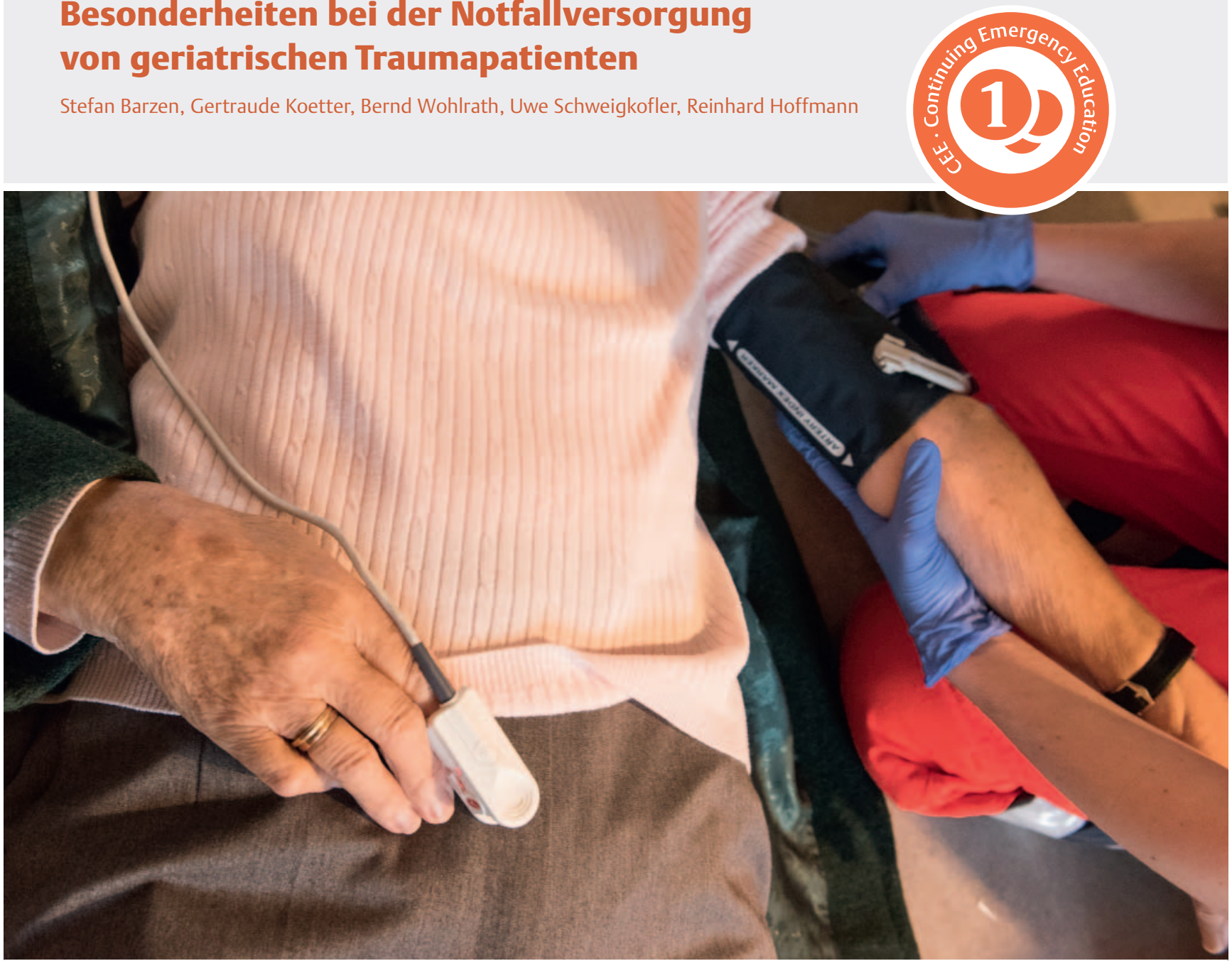

Quelle: KH Krauskopf

Die Zahl notfallmedizinischer Versorgungen geriatrischer Patienten wird aufgrund des demografischen Wandels in ihrer Bedeutung im rettungsdienstlichen Alltag zunehmen. Dieser Artikel soll eine Übersicht über die besonderen Gegebenheiten bei traumatologischen Notfällen bei diesen Patienten geben.

\begin{tabular}{|c|c|}
\hline \multicolumn{2}{|c|}{ ABKÜRZUNGEN } \\
\hline$A B C D$ & $\begin{array}{l}\text { Sicherung und Wiederherstellen } \\
\text { der Vitalfunktionen; Akronym A: } \\
\text { Airways - B: Breathing - C: Circula- } \\
\text { tion - D: Disability - E: Exposure/ } \\
\text { Environment }\end{array}$ \\
\hline AMLS & Advanced Medical Life Support \\
\hline ATLS & Advanced Trauma Life Support \\
\hline CCT & $\begin{array}{l}\text { kraniale Computertomografie } \\
\text { (Schädel-CT) }\end{array}$ \\
\hline
\end{tabular}

$\begin{array}{ll}\text { COPD } & \begin{array}{l}\text { Chronic Obstructive Pulmonary } \\ \text { Disease (chronisch obstruktive } \\ \end{array} \\ & \text { Lungenerkrankung) } \\ \text { GCS } & \text { Glasgow Coma Scale } \\ \text { HF } & \text { Herzfrequenz } \\ \text { HWS } & \text { Prehospital Trauma Life Support } \\ \text { PHTLS } & \text { Blutdruck } \\ \text { RR } & \text { Schädel-Hirn-Trauma } \\ \text { SHT } & \text { vesikuläres Atemgeräusch }\end{array}$




\section{Einleitung}

Die notfallmedizinische Versorgung geriatrischer Patienten stellt eine immer häufigere Aufgabe des rettungsdienstlichen Alltags dar und ihre Zahl wird aller Voraussicht nach weiter wachsen [1]. Darüber hinaus findet eine zunehmende Spezialisierung der Medizin im Sinne der Geriatrie statt, die sich mit den besonderen Herausforderungen der älteren Patienten beschäftigt. Dieser Artikel soll eine Übersicht über die besonderen Gegebenheiten bei der Versorgung von geriatrischen Notfallpatienten geben.

\section{Epidemiologie}

Im Jahr 2014 betrug die Zahl der über 65-Jährigen bereits 17,1 Millionen, entsprechend einem Anteil von $21 \%$ an der bundesdeutschen Gesamtbevölkerung. 2034 wird ein Anteil von 30 \% erwartet [1]. Während der Gesamtanteil an Sturzursachen bei schweren Traumata bei unter 55-Jährigen bei bis zu 23 \% liegt, zeigt die Gruppe von über 75-Jährigen einen Anteil von 76\% [2].

10 - 15\% der Behandlungen von Patienten aller Altersgruppen in Notaufnahmen sind sturzbedingt. Alte Patienten zeigen nach solchen Verletzungen ein schlechteres klinisches Outcome als jüngere Personen. Stürze stellen die Hauptursache für traumabedingte Sterbefälle bei geriatrischen Patienten dar [3-7].

\section{FALLBEISPIEL}

\section{Auffindesituation}

Die 86-jährige weibliche Patientin, Sturz in der Wohnung unklarer Genese, wird durch den Ehemann in den frühen Morgenstunden vorgefunden. Die Rettungsdienstalarmierung war bei immobilisierenden Schmerzen über den vorhandenen Hausnotruf ausgelöst worden ( $\triangleright$ Abb. 1).

\section{Definition des geriatrischen Patienten}

Die europäischen geriatrisch-medizinischen Gesellschaften definieren geriatrische und somit alterstraumatologische Patienten wie in der Infobox zusammengefasst [8].

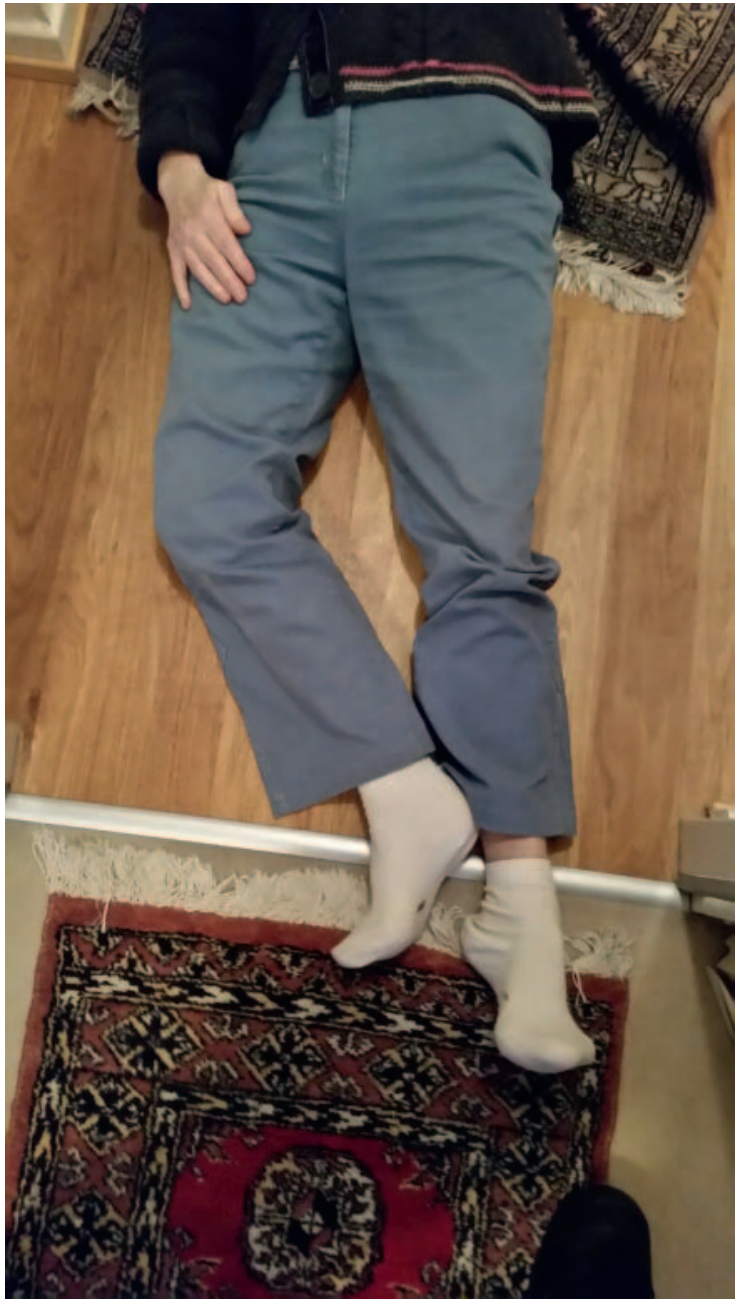

Abb. 1 Fallbeispiel: Auffindesituation [Quelle: [8] Barzen S et al. Der Sturz im Alter - Geriatrische Traumatologie in der Notfallmedizin. Notfallmedizin up2date 2016; 11: 195 - 207. doi:10.1055/s-0042-107687].

\section{DEFINITION}

\section{Geriatrischer Patient}

Die europäischen geriatrisch-medizinischen Gesellschaften definieren geriatrische Patienten folgendermaßen [8]:

- höheres Lebensalter (meist über 70 Jahre) und

- altersbedingte Funktionseinschränkungen

- Multimorbidität

oder

- Alter über 80 Jahre

- alterstypisch erhöhte Vulnerabilität

- Gefahr der Chronifizierung

- deutlich erhöhtes Risiko eines Verlusts der Autonomie mit Verschlechterung des Selbsthilfestatus 


\section{Besonderheiten des geriatrischen} Patienten

\section{Multimorbidität}

Die Errungenschaften der modernen Medizin führten und führen zu einer kontinuierlichen Verlängerung der Lebenserwartung in den westlichen Ländern. Damit geht eine zunehmende Multimorbidität der Patienten einher. Die krankheitsbezogene Mortalität sinkt, jedoch liegen zusehends mehrere therapierte Diagnosen vor [10] (s.a. Infobox Hintergrundwissen).

\section{HINTERGRUNDWISSEN}

\section{Besonderheiten geriatrischer Patienten}

- Patienten älter als 65 Jahre weisen zu 80 \% eine chronische Erkrankung auf.

- In der Altersgruppe der 70- bis 90-Jährigen liegen meist 5-10 Dauerdiagnosen vor.

- Typische Komorbiditäten sind [11, 12]:

- kardiovaskuläre Erkrankungen

- Diabetes mellitus

- COPD

- Nierenfunktionsstörungen

- demenzielle Erkrankungen

\section{Polypharmazie und Pharmakologie}

Aufgrund der Komorbiditäten erfolgt häufig eine Pharmakotherapie. Nicht selten nehmen bei leitliniengerechter Therapie geriatrische Patienten 10 oder mehr gleichzeitig verabreichte Wirkstoffe ein [13].

Merke

Im Durchschnitt nehmen geriatrische Patienten

$>6$ Medikamente regelmäßig ein.

Schätzungen zufolge verdoppelt sich das Risiko unerwünschter Arzneimittelwirkungen bei Anwendung von 4 Medikamenten gegenüber der einzelnen Substanz, bei gleichzeitiger Anwendung von 7 Wirkstoffen wird eine 14-fache Erhöhung angenommen [14].

Altersbedingte Veränderungen der Physiologie verändern die Pharmakokinetik und -dynamik. Insbesondere die Reduktion der Nierenfunktion, aber auch eine Reduktion des Extravasalvolumens und eine häufig bestehende Hypoalbuminämie nehmen Einfluss auf die Pharmakokinetik. Insgesamt kommt es zu einer Erhöhung des Fettanteils bei Reduktion des Wasseranteils. Lipidlösliche Substanzen haben somit ein größeres Verteilungsvolumen, hydrophile Substanzen ein geringeres. Dies führt z. B. zu einer Verlängerung der Eliminationszeit von Diazepam.
Pharmakodynamisch finden sich sowohl erhöhte als auch verminderte Empfindlichkeiten sowie gestörte Regulationsmechanismen und eine Häufung paradoxer Arzneimittelwirkungen. [12]

\section{PRAXIS}

Tipp

Aufgrund der vorliegenden Polypharmazie sollte eine grobe Erhebung der eingenommenen Medikamente auch schon präklinisch erfolgen. Im Idealfall sind Medikamentenlisten und/oder aktuelle Behandlungsberichte des Patienten verfügbar. Alternativ kann auch eine Pflegedokumentation oder ein pflegerischer Überleitungsbogen die gewünschten Informationen enthalten.

\section{FALLBEISPIEL}

Anamnese

Als Vorerkrankungen werden laut Pflegeakte eine arterielle Hypertonie, eine koronare Herzkrankheit und ein Vorhofflimmern angegeben. Darüber hinaus Zustand nach Hüftprothesenimplantation links vor über 10 Jahren. Beginnende leichte Demenz laut Ehemann.

Die Dauermedikation besteht aus 7 verschiedenen Präparaten, die aktuellen Dosierungen sind aus den vorliegenden Dokumenten nicht ersichtlich.

\section{Sturzneigung und -ursachen}

Es wird unterschieden zwischen

- extrinsischen, in der Umwelt begründeten, Sturzursachen und

- intrinsischen, im Patienten begründeten Sturzursachen.

Zu den extrinsischen Sturzzrsachen zählen klassischerweise

- Stolperstürze in häuslicher Umgebung, z. B. aufgrund von

- Stolperfallen,

- glatten Bodenbelägen.

Intrinsische Sturzursachen können sein:

- plötzliche Erkrankungen wie Apoplex oder Myokardinfarkt, aber auch

- psychische Veränderungen wie

- Angst,

- Unruhe,

- Demenz. 
90 \% der Stürze im Alter sind multifaktoriell bedingt, d. h. durch eine Kombination aus extrinsischen und intrinsischen Risikofaktoren. Risikofaktoren sind z. B.

- hohes Alter,

- weibliches Geschlecht,

- Pflege- und Hilfebedürftigkeit im Alltag,

- vorangegangener Sturz,

- Gangstörung,

- Gebrauch eines Hilfsmittels beim Gehen,

- Visusminderung,

- Morbus Parkinson,

- Einnahme von Psychopharmaka.

Lediglich 10 \% der Stürze sind monokausal, z. B. im Rahmen einer akuten Krankheitsattacke oder mit alleinigem extrinsischem Faktor (z. B. Glatteis). Mit zunehmendem Patientenalter steigt der Anteil intrinsischer Sturzursachen an [15].

\section{Patientenverfügung}

Merke

Das mögliche Vorliegen einer Patientenverfügung und/oder einer Vorsorgevollmacht sollte in jedem Fall erfragt werden.

Die hier als Bevollmächtige in der Gesundheitsfürsorge benannten Personen können eine wesentliche Hilfe in der Entscheidungsfindung bieten. Entsprechende Dokumente sollten, wenn möglich, in die Klinik mitgenommen werden. Darüber hinaus stellen anwesende Angehörige eine wichtige Hilfe dar, wenn es darum geht, den vermutlichen Willen des Patienten zu erörtern, auch wenn keine schriftlich fixierte Patientenverfügung vorliegt.

\section{Alterstraumatologische Zentren}

Zur Verbesserung der Qualität der Versorgung geriatrischer Traumapatienten etablieren sich vermehrt zertifizierte alterstraumatologische Zentren. Diese garantieren eine enge Zusammenarbeit zwischen Geriatrie und Unfallchirurgie und sollten bei der Wahl der Zielklinik bedacht werden.

\section{Grundlagen des praktischen Vorgehens am Unfallort}

Zur Erstuntersuchung kann die Anwendung standardisierter prioritätenorientierter Untersuchungsmethoden, z. B. nach Prehospital Trauma Life Support (PHTLS) für Traumapatienten oder nach AMLS bei internistischen Notfallbildern, hilfreich sein [16].
- Tab. 1 Primary Survey nach PHTLS und ATLS.

\begin{tabular}{|l|l|l|}
\hline Kürzel & Maßnahme & \\
\hline A & $\begin{array}{l}\text { Airway and C-Spine } \\
\text { Protection }\end{array}$ & $\begin{array}{l}\text { Atemweg sichern } \\
\text { HWS-Stabilisierung }\end{array}$ \\
\hline B & Breathing & Beatmung, Ventilation \\
\hline C & Circulation & Kreislauf \\
\hline D & Disability & neurologischer Status \\
\hline E & Exposure/Environment & $\begin{array}{l}\text { Entkleiden } \\
\text { Wärmeerhalt } \\
\text { Sturzgenese }\end{array}$ \\
\hline
\end{tabular}

\section{Primary Survey}

Im Rahmen des Primary Survey erfolgt zunächst eine strukturierte Untersuchung des Patienten nach ABCDESchema ( $\triangleright$ Tab. 1): Punkt für Punkt erfolgt die Untersuchung nach dem ABCDE-Schema, und bei vorliegenden Problemen wird zunächst die entsprechende Therapie durchgeführt, bevor das nächste Item abgearbeitet wird. Dadurch soll eine rasche, effiziente und zielorientierte Therapie akut lebensbedrohlicher Zustände erzielt werden.

\section{Secondary Survey}

Nach Ausschluss oder Behandlung einer vitalen Bedrohung des Patienten erfolgt ein Secondary Survey. Dabei wird der Unfallverletzte mittels gründlichen Bodychecks untersucht und eine ausführliche Anamnese erhoben.

Bei schweren Traumata mit akuter Lebensgefahr darf dieser ergänzende Untersuchungsschritt keine Verzögerungen bezüglich der Logistik des Transports und der Suche der Zielklinik verursachen.

\section{Cave}

Die tatsächliche Verletzungsschwere bei älteren Patienten wird leicht unterschätzt, und es erfolgt seltener als bei jüngeren Patienten eine direkte Zuweisung in ein angemessenes Traumazentrum [6].

\section{FALLBEISPIEL}

\section{Untersuchung}

Die Untersuchung erfolgt nach dem ABCDE-Schema:

- A:

- HWS frei,

- keine Schmerzhaftigkeit,

- freie Atemwege.

- B:

- VAG beidseitig,

- gute Oxygenierung.

- C:

- V.a. Schenkelhalsfraktur rechts, 
- RR und HF stabil.

- Etablierung eines i. v. Zugangs.

- D:

- GCS 15, bekannte Demenz, allseits orientiert.

- Kein sensomotorisches Defizit.

- E:

- Keine Besonderheiten.

- Sturz über eine Teppichkante (extrinsische Ursache).

\section{Besonderheiten der präklinischen Diagnostik}

\section{Anamnese}

Die Anamneseerhebung kann sich aufgrund bestehender Vorerkrankungen als stark erschwert darstellen. In jedem Fall sollte auf das Tragen von Seh- und Hörhilfen geachtet werden, um eine möglichst gute Verständigung zu ermöglichen.

Die subjektive Wahrnehmung der Beschwerden kann deutlich eingeschränkt sein, einerseits infolge ausgeschütteter Stresshormone, andererseits aufgrund vorbestehender demenzieller/neurologischer Erkrankungen oder Stoffwechselentgleisungen.

Wenn möglich, soll eine ergänzende Fremdanamnese durch Angehörige oder Pflegepersonal erfolgen. Vorhandene Pflegedokumentationen, eine Medikamentenliste und alte Arztbriefe sollten, wenn möglich, in die Klinik mitgenommen werden, um möglichst alle Nebendiagnosen auch im weiteren Therapieverlauf erfassen zu können.

Cave

Insbesondere Patienten mit demenziellen Erkrankungen stellen dabei eine Herausforderung dar. Trotz der möglicherweise eingeschränkten Aussagekraft dürfen angegebene Symptome oder Schmerzäußerungen nicht bagatellisiert werden.

\section{Präklinische Untersuchung}

Im Rahmen des Secondary Survey sind eine Inspektion und ein Bodycheck von Kopf bis Fuß unter optimierten Bedingungen (ausreichende Lichtverhältnisse, Möglichkeit der Entkleidung ohne Wärmeverlust) anzustreben. Die Entkleidung muss, wenn erforderlich, schonend und, soweit möglich, schmerzfrei erfolgen.

\section{Merke}

Insgesamt weisen geriatrische Patienten auch bei Traumata eher generelle Symptome auf.
Neben den üblichen Stabilitätstests sollte auf frische und alte Hämatome geachtet werden, da diese sowohl Anzeichen einer Sturzneigung sein können, aber auch auf eine bestehende Antikoagulation und somit ein erhöhtes Blutungsrisiko hinweisen können.

Stehende Hautfalten und eine trockene Zunge sprechen für einen schlechten Volumenstatus des Patienten und weisen auf eine möglicherweise eingeschränkte Kompensationsfähigkeit bei weiterem Volumenverlust hin.

Cave

Aufgrund der im Alter häufigen Osteoporose sind Frakturen und deren mögliche, insbesondere blutungsbedingte Komplikationen häufiger zu erwarten.

Merke

Eine kontinuierliche Überwachung der Vitalparameter ist anzustreben.

\section{Präklinische Maßnahmen}

Die präklinische Therapie geriatrischer Patienten sollte mit einem besonderen Augenmerk auf die möglicherweise vorliegenden Einschränkungen der Patienten erfolgen.

Cave

Grundsätzlich bestehen geringere Kompensationsmöglichkeiten bei kritischen Erkrankungen, andererseits wird der Zustand der Patienten aus unserer Sicht leicht unterschätzt.

In jedem Fall sollten die üblichen Basismaßnahmen sorgfältig durchgeführt werden. Eine frühzeitige, dem Notfallbild gerechte Lagerung und ein Wärmeerhalt sind anzustreben, da im Vergleich zum jüngeren Patienten ein rascherer Temperaturverlust zu erwarten ist.

Eine Notarztalarmierung sollte nach dem ortsspezifisch vorliegenden Indikationskatalog erfolgen, ebenso sollten erweiterte Maßnahmen in Abhängigkeit vom Rettungsdienstbezirk vorgenommen werden.

Bei der Medikamentenapplikation sollte nicht außer Acht gelassen werden, dass geriatrische Patienten vermehrt Nebenwirkungen oder auch paradoxe Reaktionen zeigen können. Eine entsprechende Vorbereitung bei den durch den ärztlichen Leiter Rettungsdienst freigegebenen Medikamenten ist unerlässlich. 


\section{FALLBEISPIEL}

\section{Rettungsdienstliche Erstmaßnahmen}

Der Rettungsdienst lagert die Patientin mittels Schaufeltrage ( $\triangleright$ Abb. 2 ) auf einer Vakuummatratze. Aufgrund der ausgeprägten Schmerzen ist die Notarztnachalarmierung notwendig. Im Rahmen der erweiterten Maßnahmen Gabe von 4 mg Piritramid. Erweiterung der Analgosedierung durch den Notarzt mit weiteren $6 \mathrm{mg}$ Piritramid titriert und $1 \mathrm{mg}$ Midazolam.

\section{PRAXIS}

\section{Besonderheiten der Analgosedierung und Narkose}

Die Notwenigkeit einer Analgesie scheint leicht unterschätzt zu werden. Als Beispiel sei der Transport eines Patienten mit Schenkelhalsfraktur ohne Schmerzmedikation in die Klinik genannt. Möglicherweise spielt dabei eine geringere emotionale Befangenheit des medizinischen Personals als z. B. bei kindlichen Notfällen eine Rolle.

$\rightarrow$ Eine adäquate Analgesie stellt auch beim geriatrischen Patienten ein absolutes Muss dar.

Opioide

Geriatrische Patienten weisen eine erhöhte Sensibilität gegenüber der Verabreichung von Opioiden auf und zeigen eine erhöhte Rate von z. B. Atmungskomplikationen.

$\rightarrow$ Es sollte daher eine langsame Titration bis zum gewünschten Wirkniveau erfolgen.

Hypnotika

Aufgrund einer verminderten neuronalen Dichte bei herabgesetztem zerebralem Metabolismus besteht eine erhöhte Empfindlichkeit; bei jedoch gleichzeitig herabgesetztem Herzzeitvolumen ergibt sich eine verlangsamte Anflutung. Dies kann leicht zu Überdosierungen mit konsekutiver Kreislaufdepression führen.

$\rightarrow$ Eine langsame Injektion kann daher zur Vermeidung unerwünschter Nebenwirkung hilfreich sein.

\section{Muskelrelaxanzien}

Depolarisierende Muskelrelaxanzien (z. B. Succinylcholin) weisen einen nahezu gleichen Effekt wie beim jungen Patienten auf, jedoch kann durch die Kaliumfreisetzung eine möglicherweise bestehende Hyperkaliämie verschlechtert werden.

Nicht depolarisierende Muskelrelaxanzien (z. B. Rocuronium) zeigen eine gegenüber jüngeren Personen verminderte Anflutung bei verlängerter Elimination [17 - 19].

Im Folgenden sind spezielle Verletzungsmuster beschrieben.

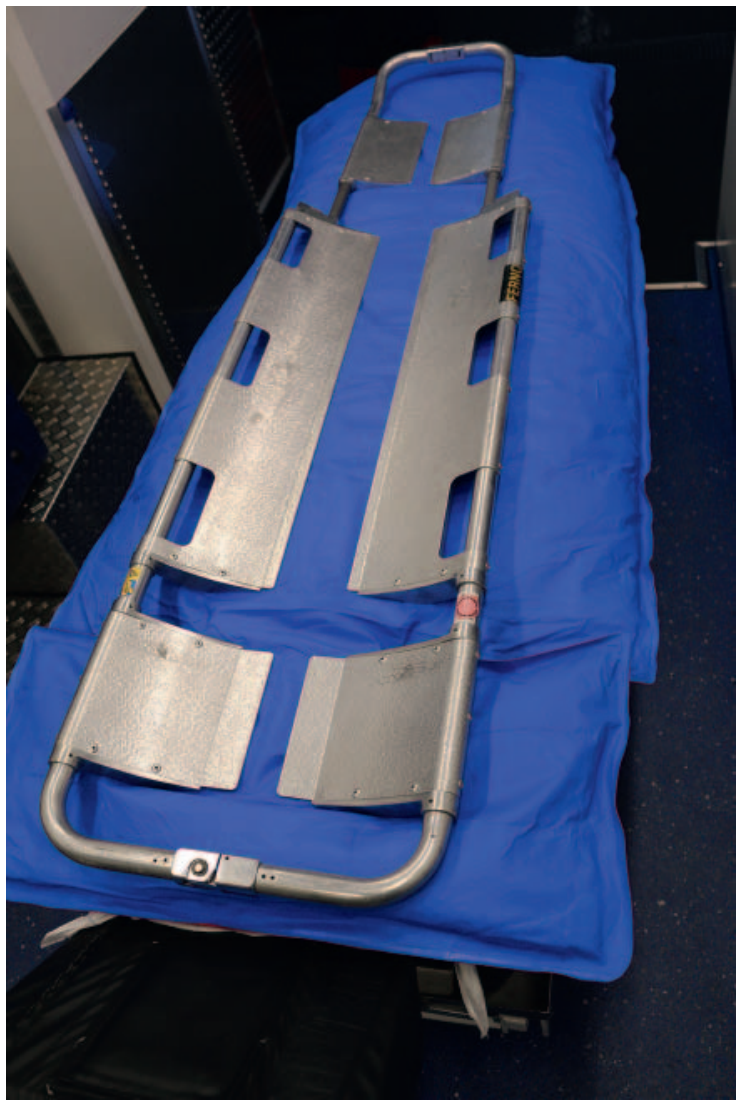

- Abb. 2 Schaufeltrage [Quelle: [8] Barzen S et al. Der Sturz im Alter - Geriatrische Traumatologie in der Notfallmedizin. Notfallmedizin up2date 2016; 11: 195- 207. doi:10.1055/s-0042-107687].

\section{Schädel-Hirn-Trauma (SHT)}

Verletzungen im Bereich des Schädels und des Gesichtsschädels stellen häufige Sturzfolgen bei geriatrischen Patienten dar. Als klassische Verletzung sei die RissQuetsch-Wunde im Stirn-/Augenbrauenbereich genannt.

Eine mögliche Antikoagulation muss erfragt werden, da diese Medikation eine erhöhte Rate von intrakraniellen Blutungen bedingt.

Merke

Wenn eine Antikoagulation nicht zu erheben ist, muss von einer bestehenden Antikoagulation ausgegangen werden.

\section{PRAXIS}

\section{Diagnostische Abklärung}

Auch ohne Hautwunde, ohne Amnesie und adäquate Glasgow Coma Scale sollte somit in jedem Fall nach einem Sturz eine Abklärung im Krankenhaus - wenn realisierbar mit Möglichkeit der Durchführung einer kranialen Computertomografie (CCT) - erfolgen. 
Im Allgemeinen zeigen Patienten mit einem Alter > 81 Jahre eine erheblich erhöhte Mortalität bei jedem erhobenen initialen GCS [4].

Die Sicherung der Vitalparameter steht zunächst im Vordergrund, bei vermeintlicher Kompromittierung der Schutzreflexe wird eine präklinische Narkose empfohlen.

\section{Frakturen}

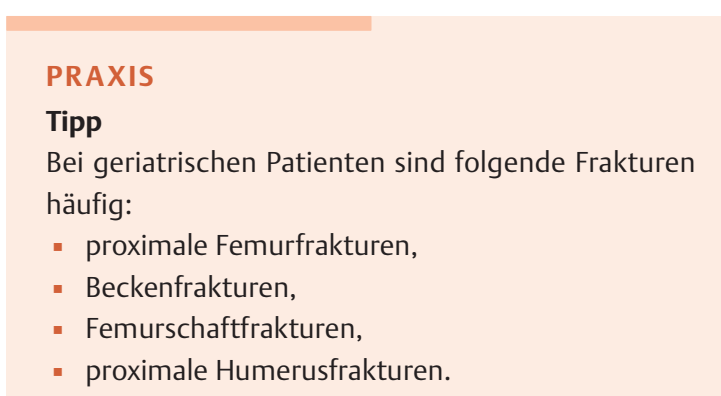

Es sollte immer gezielt daraufhin untersucht werden.

Auf die Gesamtzahl der vorliegenden Frakturen bezogen führen proximale Femurfrakturen vor distalen Radiusfrakturen und proximalen Humerusfrakturen.

Aufgrund der im Alter häufig vorbestehenden Osteoporose treten in dieser Altersgruppe insgesamt häufiger Frakturen auf als bei jüngeren Personen [20]. Des Weiteren wird die Zahl periprothetischer Frakturen erwartungsgemäß weiter zunehmen. Eine Ruhigstellung kann sich daher unter Umständen als deutlich erschwert erweisen.

Die Höhe des Blutverlustes bei geschlossenen Frakturen zeigt $>$ Tab. 2.

\section{Proximale Femurfraktur}

Die beim alten Menschen nach Sturzereignissen häufigste Frakturentität stellt die proximale Femurfraktur dar.

Neben der üblicherweise bestehenden ausgeprägten Schmerzsymptomatik, in der klinischen Untersuchung anhand von Leisten- und Trochanterklopfschmerz reproduzierbar, steht als wegweisendes Zeichen die Verkürzung des Beines bei Außenrotationsfehlstellung im Vordergrund. Bei nur gering dislozierten pertrochantären Frakturen kann diese jedoch nur leicht ausgeprägt sein.

Eine achsgerechte Ruhigstellung sollte zum einen zur Schmerztherapie, zum anderen bei nicht auszuschließenden Begleitfrakturen, insbesondere des Beckens und der Wirbelsäule, in jedem Fall erfolgen. Zur Ruhigstellung bietet sich die Immobilisation mittels Vakuummatratze nach entsprechend schonender Umlagerung unter An-
- Tab. 2 Blutverlust bei geschlossenen Frakturen.

\begin{tabular}{|l|l|}
\hline $\begin{array}{l}\text { Frakturlokalisation } \\
\text { (geschlossene Fraktur) }\end{array}$ & Blutverlust \\
\hline Radius/Ulna & $50-400 \mathrm{ml}$ \\
\hline Humerus & $100-800 \mathrm{ml}$ \\
\hline Femur & $300-2000 \mathrm{ml}$ \\
\hline Becken & $500-5000 \mathrm{ml}$ \\
\hline
\end{tabular}

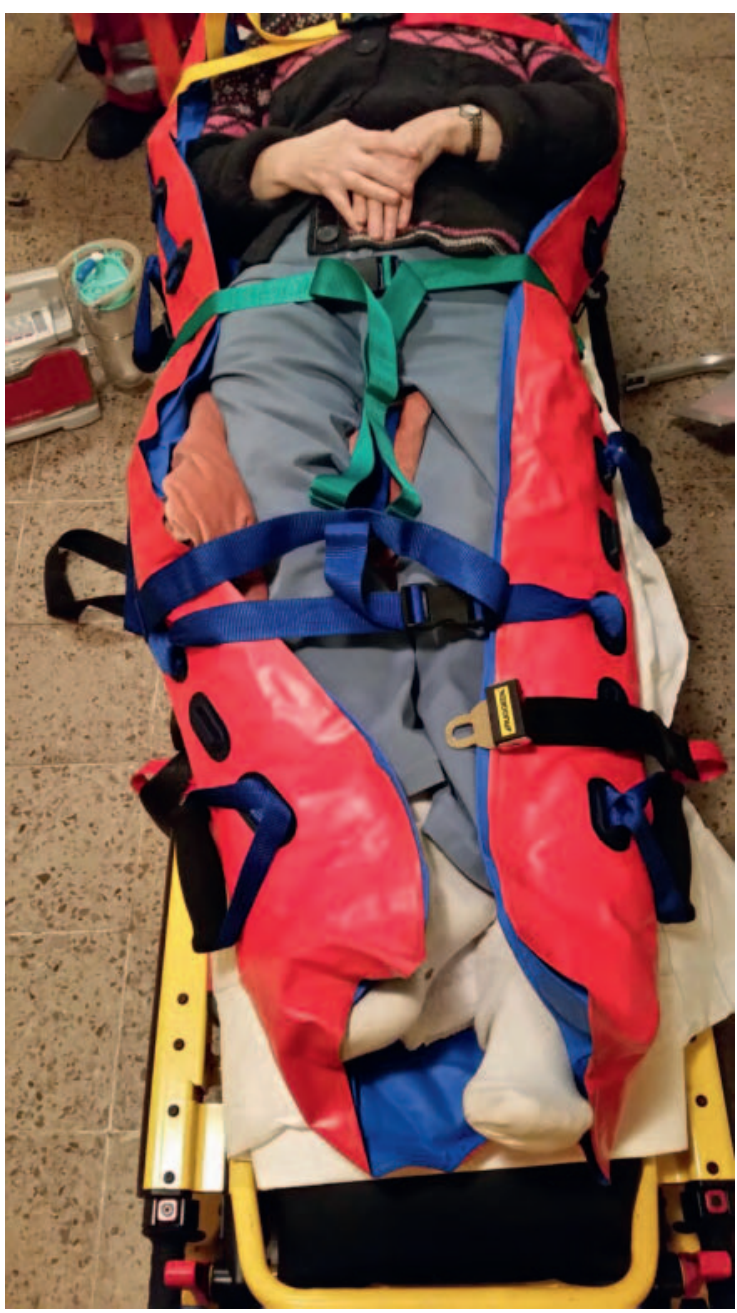

Abb. 3 Fallbeispiel: Ruhigstellung mittels Vakuummatratze [Quelle: [8] Barzen S et al. Der Sturz im Alter - Geriatrische Traumatologie in der Notfallmedizin. Notfallmedizin up2date 2016; 11: 195 - 207. doi:10.1055/s-0042107687].

wendung einer Schaufeltrage ( $\mathbf{A} \mathbf{b} \mathbf{b} . \mathbf{2}, \mathbf{3}$ ) und ggf. nach vorher erfolgter Anlage einer Halsorthese (z. B. Stifneck) an. Steht diese nicht zur Verfügung, ist auch eine adäquate Lagerung auf dem Spineboard erlaubt. 


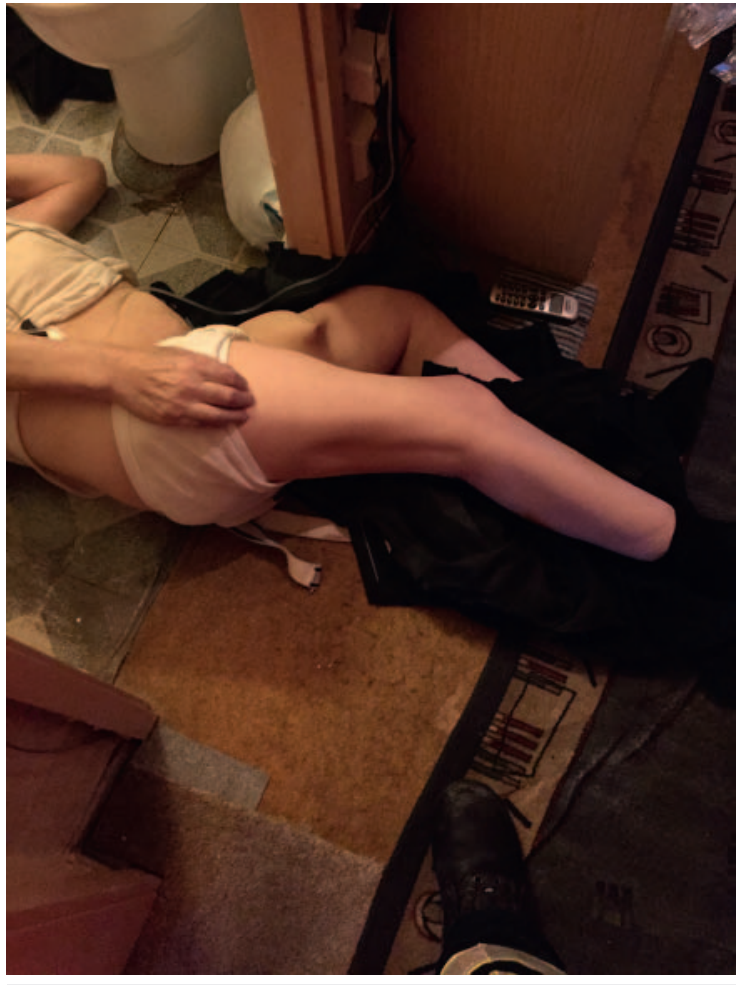

- Abb.4 Auffindesituation periprothetische Femurfraktur ante perforationem [Quelle: [8] Barzen S et al. Der Sturz im Alter - Geriatrische Traumatologie in der Notfallmedizin. Notfallmedizin up2date 2016; 11: 195 - 207. doi:10.1055/s-0042-107687].

\section{FALLBEISPIEL}

\section{Immobilisierung und Kliniktransport}

Abschließende Ruhigstellung in Vakuummatratze

( $\vee$ Abb. 3) und Transport in die Klinik.

\section{PRAXIS}

\section{Proximale Femurfraktur}

Meist zeigt sich die proximale Femurfraktur an der Verkürzung und Außenrotation des betroffenen Beins, die jedoch nicht ausgeprägt vorliegen muss. Eine schonende Lagerung mittels Schaufeltrage und Vakuummatratze hat sich bewährt.

In jedem Fall sollte eine ausreichende Analgesie erfolgen.

\section{Periprothetische Frakturen}

Prothesenassoziierte Frakturen stellen eine Besonderheit dar. Dennoch wird eine zunehmende Häufigkeit dieser Verletzungen erwartet. Periprothetische Frakturen können eine Herausforderung im Rettungsdienst darstellen aufgrund
- der häufig erschwerten Ruhigstellung,

- der nicht unerheblichen Blutungsgefahr (bei z. B.

Knochenfragmenten ante perforationem) und

- der unter Umständen ausgedehnten Weichteilschäden.

\section{HINTERGRUNDWISSEN}

Lokalisationen periprothetischer Frakturen (in absteigender Häufigkeit)

- proximale prothesennahe Femurfrakturen $(\triangleright$ Abb. 4)

- distale Femurfrakturen

- proximale Tibiafrakturen

- proximale Humerusfrakturen

Zur Ruhigstellung eignet sich im Bereich der unteren Extremität meist die Vakuummatratze, im Bereich der oberen Extremität ein Dreiecktuch.

\section{KERNAUSSAGEN}

\section{Geriatrische Traumapatienten}

Epidemiologie

Die Häufigkeit von Rettungsdiensteinsätzen bei geriatrischen Patienten wird weiter steigen. Das Wissen um die Besonderheiten dieser Patientengruppe wird somit immer wichtiger.

Definition geriatrischer Patient

- Alter:

- Multimorbidität und Alter über 70 Jahre oder

- Alter über 80 Jahre mit entsprechend erhöhter Vulnerabilität.

- Polypharmazie und veränderte Pharmakodynamik und -kinetik.

- Mehrfachtherapie mit erhöhten Nebenwirkungen erschwert die Therapie.

- Aufgrund der verlangsamten Stoffwechselprozesse Gefahr der Überdosierung von Medikamenten!

\section{Anamnese}

- Erschwerte Anamneseerhebung bei demenziell vorerkrankten Patienten.

- Achten auf das Tragen von Seh- und Hörhilfen.

Untersuchung

- Symptome können atypisch präsentiert werden.

- Kein Bagatellisieren der dargebotenen Beschwerden.

Therapie

Die Analgosedierung hat ein erhöhtes Nebenwirkungspotenzial, darf jedoch in keinem Fall vernachlässigt werden. 
Frakturen

- Häufigste Fraktur ist die proximale Femurfraktur.

- Periprothetische Frakturen stellen bisher eine Seltenheit dar, ihre Zahl wird aber weiter zunehmen.

- Adäquate Ruhigstellung und Schienung sind unbedingt erforderlich.

Interessenkonflikt

Die Autoren geben an, dass kein Interessenkonflikt besteht.

\section{Autorinnen/Autoren}

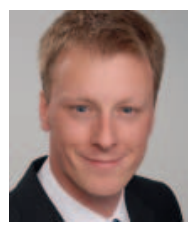

\section{Dr. med. Stefan Barzen}

Bis zur Beendigung des Studiums als Rettungsassistent tätig. Oberarzt der Abteilung für Unfallchirurgie und orthopädische Chirurgie der BG Unfallklinik Frankfurt am Main. Stellvertretender Ärztlicher Leiter RTH Christoph 2 und NEF 1. Notarzt auf dem NEF 1 und RTH Christoph 2 (BG Unfallklinik Frankfurt am Main).

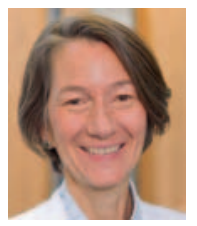

\section{Gertraude Koetter}

Oberärztin der Klinik für Geriatrie des Agaplesion Markus Krankenhauses in Frankfurt am Main. Führende geriatrische Betreuung des alterstraumatologischen Zentrums der BG Unfallklinik Frankfurt am Main.

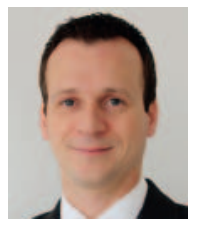

\section{Dr. med. Bernd Wohlrath}

Oberarzt der Abteilung für Unfallchirurgie und orthopädische Chirurgie der BG Unfallklinik Frankfurt am Main. Ärztlicher Leiter RTH Christoph 2 und NEF 1. Notarzt auf dem NEF 1 und RTH Christoph 2 (BG Unfallklinik Frankfurt am Main).

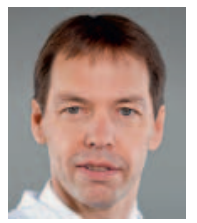

\section{Dr. med. Uwe Schweigkofler}

Stv. Ärztlicher Direktor der BG Unfallklinik Frankfurt am Main, Notarzt auf dem RTH Christoph 2, Leitender Notarzt der Stadt Frankfurt am Main.

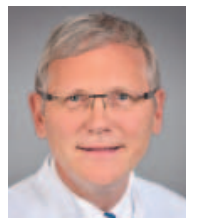

\section{Prof. Dr. med. Dr. med. habil. Reinhard Hoffmann}

Ärztlicher Direktor und Chefarzt der Abteilung für Unfallchirurgie und Orthopädische Chirurgie der BG Unfallklinik Frankfurt am Main. Vizepräsident des BVOU

\section{Erstveröffentlichung}

Dieser Beitrag ist eine aktualisierte Version des Artikels: Barzen S, Koetter G, Wohlrath B et al. Der Sturz im Alter - Geriatrische Traumatologie in der Notfallmedizin. Notfallmedizin up2date 2016; 11: 195 - 207. doi:10.1055/s-0042-107687.1
Korrespondenzadresse

Dr. med. Stefan Barzen

Abteilung für Unfallchirurgie und Orthopädische Chirurgie Berufsgenossenschaftliche Unfallklinik Frankfurt am Main Friedberger Landstraße 430

60389 Frankfurt am Main

stefan.barzen@bgu-frankfurt.de

\section{Literatur}

[1] Statistisches Bundesamt. Koordinierte Bevölkerungsvorausberechnung für Deutschland. 04.07.2015

[2] Lukin W, Greenslade JH, Chu K et al. Triaging older major trauma patients in the emergency department: an observational study. Emerg Med J 2014; 32 (4): 281 - 286

[3] Roudsari BS, Ebel BE, Corso PS et al. The acute medical care costs of fall-related injuries among the U.S. older adults. Injury 2005; 36 (11): 1316-1322

[4] O'Neill S, Brady RR, Kerssens J] et al. Mortality associated with traumatic injuries in the elderly: a population based study. Arch Gerontol Geriat 2012; 54 (3): e426 - e430

[5] Grossman MD, Miller D, Scaff DW et al. When is an elder old? Effect of preexisting conditions on mortality in geriatric trauma. J Trauma Acute Care Surg 2002; 52 (2): 242-246

[6] Jacobs DG. Special considerations in geriatric injury. Curr Opinion Crit Care 2003; 9 (6): 535 - 539

[7] Rosen T, Mack KA, Noonan RK. Slipping and tripping: fall injuries in adults associated with rugs and carpets. J Injury Violence Res 2013; 5 (1): 61

[8] Barzen S, Koetter G, Wohlrath B et al. Der Sturz im Alter Geriatrische Traumatologie in der Notfallmedizin. Notfallmedizin up2date 2016; 11: 195-207. doi:10.1055/s-0042107687

[9] Geriatrie DGfGu. Was ist Geriatrie. Z Gerontol Geriat 2009; $42(5): 412-415$

[10] Aminzadeh F, Dalziel WB. Older adults in the emergency department: a systematic review of patterns of use, adverse outcomes, and effectiveness of interventions. Ann Emerg Med 2002; 39 (3): $238-247$

[11] Todd C, Skelton D, World Health Organization - WHO. What are the main risk factors for falls amongst older people and what are the most effective interventions to prevent these falls? Geneva: World Health Organization. 2004

[12] Berthold H, Steinhagen-Thiessen E. Arzneimitteltherapie im Alter. Internist 2009; 50 (12): 1415-1424

[13] Boyd CM, Darer J, Boult C et al. Clinical practice guidelines and quality of care for older patients with multiple comorbid diseases: implications for pay for performance. JAMA 2005; 294 (6): $716-724$

[14] Chrischilles EA, Segar ET, Wallace RB. Self-reported adverse drug reactions and related resource use: a study of community-dwelling persons 65 years of age and older. Ann intern Med 1992; 117 (8): 634-640

[15] Herrmann R, Meier-Baumgartner H. Die sturzbedingte, proximale Femurfraktur älterer Menschen - Ätiologie und Rehabilitation. Z Gerontol Geriat 1999; 32 (1): 52 - 57

[16] Bernhardt M. Präklinisches Traumamanagement: Prehospital Trauma Life Support (PHTLS). Deutsche Bearbeitung durch PHTLS Deutschland und Schweiz. Berlin: Elsevier, Urban \& Fischer; 2012 
[17] Kratz C, Schleppers A, Iber T et al. Pharmakologische Besonderheiten und Probleme des älteren Patienten. Anaesthesist 2005; 54 (5): 467-475

[18] Lamy PP. Comparative pharmacokinetic changes and drug therapy in an older population. J Am Geriat Soc 1982; 30 (Suppl. 11): S11-S19

[19] Cohendy R, Brougere A, Cuvillon P. Anaesthesia in the older patient. Current Opinion Clin Nutrit Metab Care 2005; 8 (1): $17-21$

[20] Court-Brown CM, Caesar B. Epidemiology of adult fractures: a review. Injury 2006; 37 (8): $691-697$
Bibliografie

DOI https://doi.org/10.1055/a-0648-2334 retten 2019; 8: 32-43

(c) Georg Thieme Verlag KG, Stuttgart · New York ISSN 2193-2387 


\section{Punkte sammeln auf CME.thieme.de}

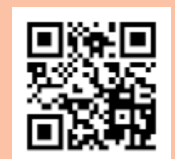

Diese Fortbildungseinheit ist in der Regel 12 Monate online für die Teilnahme verfügbar.

Den genauen Einsendeschluss finden Sie under https://eref.thieme.de/CXB4YLZ.

Sollten Sie Fragen zur Online-Teilnahme haben, finden Sie unter https://cme.thieme.de/hilfe

eine ausführliche Anleitung. Wir wünschen viel Erfolg beim Beantworten

der Fragen!

Unter https://eref.thieme.de/CXB4YLZ oder über den QR-Code kommen Sie

direkt zur Startseite des Wissenstests.

\section{Frage 1}

Wie groß ist der Prozentsatz der Patienten aller Altersgruppen, die in Notaufnahmen aufgrund von Stürzen behandelt werden?
A $2-5 \%$
B $5-10 \%$
C $10-15 \%$
D ca. $20 \%$
E $>30 \%$

\section{Frage 2}

Wie viele ärztlich verschriebene Medikamente nehmen geriatrische Patienten im Durchschnitt ein?

A Sie nehmen keine Medikamente ein.

B Sie nehmen 1 bis 2 Medikamente ein.

C Sie nehmen 3 bis 4 Medikamente ein.

D Sie nehmen 5 bis 6 Medikamente ein.

E Sie nehmen mehr als 6 Medikamente ein.

\section{Frage 3}

Welche der genannten Krankheiten stellt keine typische Komorbidität alter Patienten dar?
A Diabetes mellitus
B chronisch obstruktive Lungenerkrankung (COPD)
C demenzielle Erkrankungen
D allergisches Asthma
E kardiovaskuläre Erkrankungen

\section{Frage 4}

Wie viel Prozent der Patienten über 65 Jahre weisen eine chronische Erkrankung auf?
A $10 \%$
B $20 \%$
C $40 \%$
D $60 \%$
E $80 \%$

\section{Frage 5}

Veränderungen der Physiologie führen zur Änderung der Pharmakokinetik und-dynamik. Welche der folgenden Aussagen in diesem Zusammenhang ist falsch?

A Es kommt zu einer Zunahme paradoxer Arzneimittelwirkungen.

B Es bestehen erhöhte Empfindlichkeiten gegenüber verabreichten Medikamenten.

C Es bestehen verminderte Empfindlichkeiten gegenüber verabreichten Medikamenten

D Hydrophile Substanzen haben ein geringeres Verteilungsvolumen.

E Lipidlösliche Substanzen haben ein geringeres Verteilungsvolumen.

\section{Frage 6}

Welcher Unfallmechanismus zählt nicht zu den extrinsischen Sturzursachen?
A Sturz über Stolperfalle
B Sturz aufgrund veränderter Umgebung
C Sturz aufgrund vermehrter Unruhe und Angst
D Sturz auf glattem Bodenbelag
E Sturz über eine Teppichkante beim Toilettengang

\section{Frage 7}

Wodurch ist die Erstuntersuchung nach Prehospital Trauma Life Support (PHTLS) oder Advanced Trauma Life Support (ATLS) gekennzeichnet?

A Man beginnt mit einer körperlichen Untersuchung von Kopf bis Fuß.

B Eine Kreislaufkontrolle ist die erste Maßnahme.

C Die Unfallkinematik lässt keine Rückschlüsse auf das Verletzungsmuster zu.

D Die Erstuntersuchung erfolgt nach dem ABCDE-Schema.

E Die Kontrolle der Pupillen ist beim Schädel-Hirn-Trauma die erste Maßnahme.

- Weitere Fragen auf der folgenden Seite... 


\section{Punkte sammeln auf CME.thieme.de}

\section{Frage 8}

Im Rahmen der Analgosedierung und Narkose geriatrischer Patienten weisen die Substanzklassen alterstypische Besonderheiten auf. Welche gehört nicht dazu?

A Es besteht eine erhöhte Sensitivität gegenüber der Verabreichung von Opioiden.

B Hypnotika fluten schnell an und führen selten zu Kreislaufdepressionen.

C Eine langsame Injektion der Substanzen kann zur Vermeidung unerwünschter Nebenwirkungen führen.

D Wegen der im Alter verlangsamten Stoffwechselprozesse sollten die üblichen Erwachsenendosierungen entsprechend angepasst werden.

E Nicht depolarisierende Muskelrelaxanzien zeigen bei geriatrischen Patienten eine geringere Anflutung als bei jüngeren Menschen, bei verlängerter Elimination.

\section{Frage 9}

Welche Aussage im Zusammenhang mit Schädel-Hirn-Traumata bei geriatrischen Patienten trifft nicht zu?

A Schädel-Hirn-Traumata stellen häufige Verletzungen geriatrischer Patienten dar.

B Wenn keine Hautwunde und ein adäquater Glasgow Coma Scale vorliegen, ist eine Abklärung im Krankenhaus nicht erforderlich.

C Eine bestehende Einnahme von Antikoagulation muss kritisch gewertet werden bzgl. erhöhter Blutungskomplikationen.

D Patienten älter als 81 Jahre weisen eine deutlich erhöhte Mortalität auf bei jedem erhobenen initialen Glasgow Coma Score (GCS).

E Die Sicherung der Vitalparameter steht im Vordergrund.

\section{Frage 10}

Eine der genannten Frakturen zählt nicht zu typischen Frakturen geriatrischer Patienten. Welche?
A proximale Femurfrakturen
B Tibiakopffrakturen
C proximale Humerusfrakturen
D Beckenfrakturen
E distale Radiusfrakturen 